\title{
Icariin Attenuates Serum Deprived-Induced Premature Senescence in Rat Annulus Fibrosus Cells and Ameliorates Disc Degeneration In Vivo
}

\author{
Xiangyu Deng*, Sheng Chen, Hang Liang, Dong Zheng and Zengwu Shao \\ Department of Orthopaedic Surgery, Union Hospital, Tongji Medical College, Huazhong University of Science and Technology, \\ China
}

\begin{abstract}
Purpose: To explore the effect of icariin, a traditional chinese medicine, on serum deprived-induced premature senescence in rat annulus fibrosus cells in vitro as well as it's possible mechanism and IDD in vivo.

Methods and Results: Rat annulus fibrosus cells were separated from intervertebral disc of $200 \mathrm{~g}$ rat with sterile surgical instruments and they were used for all experiments after 3 passages. The rat annulus fibrosus cells were divided into three groups: A (blank control: 10\% fetal bovine serum of F12 DMEM); B (2\% fetal bovine serum of F12 DMEM); C ( $2 \%$ fetal bovine serum of F12 DMEM + $20 \mu \mathrm{M}$ icariin). We detected cell proliferation, SA- $\beta$-Gal activity, cell cycle, and the expression of matrix macromolecules (aggrecan and collagen I) and senescence markers (p16 and p53). All results supported each other and showed a remarkable protect effect of icariin which can attenuate serum deprived-induced premature senescence in rat annulus fibrosus cells in vitro. We also found the variation of ROS/NF- $\mathrm{KB}$ pathway which indicated that ROS/NF- $\mathrm{BB}$ pathway was at least partly included in this protect process. Meanwhile, in a rat model, we observed protect effect when rat accept the intervention of icariin intraperitoneal infection every other day. These protect effect have been verified by HE stains and immunohistochemistry stain.
\end{abstract}

Conclusion: These results reveal that icariin could protect against serum deprived-induced premature senescence of rat NP cells through ROS/NF- $\kappa \mathrm{B}$ pathway and ameliorate disc degeneration in vivo and suggest that icariin can be a new therapeutic candidate for treatment of IDD.

KEYWORDS: Icariin; Annulus fibrosus cells; ROS/NF-кB pathway; Premature senescence; Serum deprived

ABBREVIATIONS: IDD: Intervertevral Disc Degeneration; ROS: Reactive Oxygen Species; NF-кB: Nuclear factor kappa-B; NP: Nucleus Puplosus; AF: Annulus Fibrosus; SA- $\beta$-Gal: Senescence- associated $\beta$ - galactosidase

\section{INTRODUCTION}

Degeneration of intervertebral disc is a worldwide degenerative disease and brings us large load of economic burden [1,2]. Regeneration and repair of intervertebral disc is vital. Annulus fibrosus senescence is important in this pathological process [3]. Research showed annulus fibrosus is different in young and old sample using intralamellar matrix of single lamellae [4], Quantitative proteomic analysis [5] and medical imaging methods [6]. Although death and reduction of extracelluar matrix in nucleus pulposus cells contribute to the degeneration of intervertebral disc [7], the senescence and fracture of annulus fibrosus is cannot be neglected.
Quick Response Code:

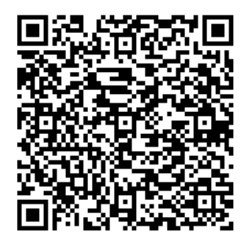

Address for correspondence: Xiangyu Deng, Department of Orthopaedic Surgery, Union Hospital, Tongji Medical College, Huazhong University of Science and Technology, China

Received: September 19, 2020 Published: September 23, 2020

How to cite this article: Xiangyu D, Sheng C, Hang L, Dong Z, Zengwu S. Icariin Attenuates Serum Deprived-Induced Premature Senescence in Rat Annulus Fibrosus Cells and Ameliorates Disc Degeneration In Vivo. 2020 - 2(5) OAJBS.ID.000219. DOI: 10.38125/OAJBS.000219 
Icariin is a traditional Chinese medicine and a kind of natural flavonol glycoside. Plentiful literature report icariin have effect of prevent apoptosis [8-10], attenuate inflammation [11], prolong life [12] and resistance senescence [13-18]. Nevertheless, the mechanism of resistance senescence is poorly understanding. We know intervertebral disc cells including nucleus puplosus cells (NP) and annulus fibrosus cells (AF) are nutritional deficiency in vivo for the special inter-vertebra location and blood supply deficiency. We chose serum-deprived model in vitro for simulation of nutritional deficiency in vivo. Serum-deprived have the effect of activating NF$\kappa B$ pathway have been verified [19-21] in some research and the activation of NF- $\kappa B$ is associated with senescence [22-24]. So, we explored if icariin has protected effect when AF cells are exposed to nutritional deficiency and the effect of IVDD repair in vivo.

\section{MATERIALS AND METHODS}

Cell culture and synchronization. Annulus fibrosus cells were isolated using about $200 \mathrm{~g}$ NP tissue of rats. Briefly, annulus fibrosus tissue was aseptically removed in a petri dish containing $0.25 \%$ $(\mathrm{w} / \mathrm{v})$ type I collagenase and cut into pieces $0.1 \mathrm{~mm} \times 0.1 \mathrm{~mm}$. Then samples were digested with $0.25 \%(\mathrm{w} / \mathrm{v})$ type I collagenase for 3-4h and serum was used to stop the reaction. After centrifugation at $1200 \mathrm{rpm}$ for $5 \mathrm{~min}$, the supernatant was discarded, and the pellet was resuspended in F12-Dulbecco's modified Eagle medium supplemented with $20 \%$ fetal bovine serum, $100 \mathrm{U} / \mathrm{mL}$ penicillin, and $100 \mathrm{mg} / \mathrm{L}$ streptomycin. Cell cultures were maintained at 37 ${ }^{\circ} \mathrm{C}$ and 5\% $\mathrm{CO}_{2}$. Medium was changed 3-5 days later when the cells had attached, and then changed every other day. When AF cells reached approximately $80 \%$ confluence, each primary culture was subculture at a $1: 3$ ratio with a $0.25 \%(\mathrm{w} / \mathrm{v})$ trypsin solution.

Grouping. A (blank control: 10\% fetal bovine serum of F12 DMED); B ( $2 \%$ fetal bovine serum of F12 DMEM for 48h); C ( $2 \%$ fetal bovine serum of F12 DMEM $+20 \mu \mathrm{M}$ icariin). In vivo experiment Rats were randomly divided into three groups: the control group (A), the IVDD group (B) and the icariin treated group (C). Intervertebral disc degeneration and premature senescence model was applied to the latter two groups and referred to needle puncture model [25]: Briefly, rat tail disc (Co7/8) was chosen as the experimental level. Needles (27G, $4 \mathrm{~mm}$ long) were used to puncture the whole layer of annulus fibrosus though the tail skin and kept in the disc for $1 \mathrm{~min}$. After the procedure, the rats were placed back in their cages and given food and water. And the rats in group C were intraperitoneal injected with icariin $(50 \mathrm{mg} / \mathrm{kg})$ every other day. After 8 weeks puncture, the rats was killed and tail discs (Co7/8) were analyzed. SA- $\beta$-Gal activity detection. AF cells were seeded in 6-well plates and incubated with different test compounds for 24 hours. Then, SA- $\beta$-Gal staining was performed using a Senescence $\beta$-Galactosidase Staining Kit (Beyotime, China) and the SA- $\beta$-Gal staining-positive NP cells were observed under a light microscope (Olympus BX51). SA- $\beta$-Gal activity was expressed as the percentage of SA- $\beta$-Gal positive AF cells.

Cell cycle analysis. AF cells were seeded in six well plates and grown to $60-70 \%$ confluence and with different test compounds for 24 hours. Thereafter, the AF cells were digested with trypsin $(0.25 \%$ without EDTA, Gibco) and centrifuged to collect the cell pellets. After fixation with $75 \%$ ethanol overnight, treatment with RNAse for $30 \mathrm{~min}$ in $37^{\circ} \mathrm{C}$ and staining with propidium iodide dye (50 ug/ $\mathrm{ml}$, Beyotime, China) for 30 minutes. AF cells were subjected to flow cytometry analysis. The cell cycle of each group was analyzed using multicycle software (Japan PHENIX Company).
Immunocytochemistry staining. Expression of matrix macromolecules (collagen I) and senescence related protein was analyzed by immunocytochemistry. Briefly, AF cells were first fixed with $4 \%$ paraformaldehyde and blocked with $5 \%$ bovine serum albumin. After incubation with primary antibodies (collagen I: NOVUS, NB600-450, diluted 1:1000; p53: abcam, ab26, diluted $1: 500$ ) at $4{ }^{\circ} \mathrm{C}$ overnight and incubation with the corresponding secondary antibodies conjugated with green or red fluorescence (ZSGB-BIO, China, diluted 1:2000) at $37^{\circ} \mathrm{C}$ for 2 hours. Finally, the AF cells were observed under a light microscopy (Olympus BX51, Japan) and immunostaining intensity was analyzed using ImagePro Plus software (Version 5.1, Media Cybernetics, Inc.).

Detection of intracellular ROS levels by flow cytometry: Cells were treated differently according to the experiment grouping design. Then $200 \mu \mathrm{L}$ of culture medium from each group were gathered to detect intracellular ROS levels. Experimental steps were strictly executed according to the manufacturer's instructions.

Expression of p16, p53, NF- $\kappa B$ (p-P65) by Western blot analysis: Proteins were extracted according to the instructions of the Total Extraction Sample Kit. Equal amounts of proteins (10 ug) were loaded onto $10 \%$ sodium dodecyl sulfate polyacrylamide gels, electrophoresed, and then transferred to polyvinylidene fluoride membranes. The membranes were incubated with $5 \%$ nonfat milk for $2 \mathrm{~h}$ followed by incubation with a primary antibody overnight at $4{ }^{\circ} \mathrm{C}(0.5 \mathrm{ug} / \mathrm{mL}$ p16, p53, NF- $\mathrm{B}$ (p-P65); 1:1000). After washing in TBST, membranes were incubated with the secondary antibody for $1.5 \mathrm{~h}$ at room temperature (rabbit anti-mouse or goat anti-rabbit, 1:5000). Bands were visualized by incubating with enhanced chemiluminescence reagent for 2 min after membranes were washed with TBST. Densitometry of p16, p53 as well as NF$\kappa B$ (p-P65) levels was performed using Image J software (National Institutes of Health, Bethesda, MD, USA).

Statistical analysis: Data are presented as means \pm standard deviation. For group-wise comparisons, a one-way ANOVA with the LSD or Dunnett's T3 test was performed using SPSS 19.0 (IBM, Chicago, IL, USA). Values were considered significantly different for $\mathrm{P}<0.05$.

\section{RESULTS}

Icariin reduced SA- $\beta$-Gal activity of serum-deprived AF cells. SA- $\beta$-Gal activity is an examinational mean based on increased activity of SA- $\beta$-Gal when cells get into senescence phase. SA- $\beta$ Gal can be detecting with a reaction substrate of X-Gal when $\mathrm{pH}$ is 6.0. Compared with control group (Figure $1 \mathrm{~A}$ ), serum-deprived $\mathrm{AF}$ cells change into larger volume and more SA- $\beta$-Gal activity stain (Figure 1B). Icariin attenuate this senescence change (Figure 1C). We simulate a premature senescence model using serum-deprive method and there are reasons to believe that icariin has significant protective effect when rat annulus fibrosis cells exposed in an environment of nutritional deficiency.

G0-G1 cell cycle arrest is another important characteristic of senescence cell. Cell in G0-G1 period stand for block of cell. Cell cycle was delayed in G1 period when serum was deprived compared with blank group. Icariin have apparent effect on reversing this phenomenon (Figure 2). There has statistic difference between group $B$ and group $C$ with three times repetition $\left({ }^{*} \mathrm{p}<0.01\right)$.

P53 and P16 are two classic markers of cell senescence, their protein expression was investigated to support our conclusion 
that icariin can protect against serum-deprived-induced $\mathrm{AF}$ cell senescence proteins P53 and P16 increased (Figure 3). Immunofluorescence in the cells of nucleus proteins P53 and P16 (Figure 3A), same as western blot support each other to come to a conclusion that icariin have the ability to decrease the expression level of senescence relative proteins (Figure 3B). This data has a significant statistic difference (Figure $3 C) .\left({ }^{*} \mathrm{p}<0.05,{ }^{* *} \mathrm{p}<0.01\right)$.

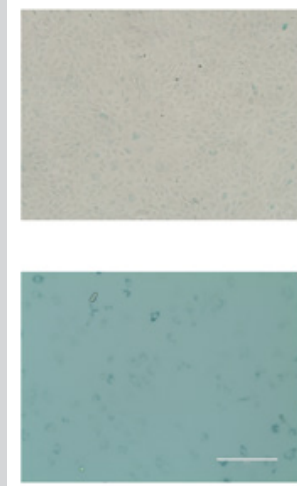

A
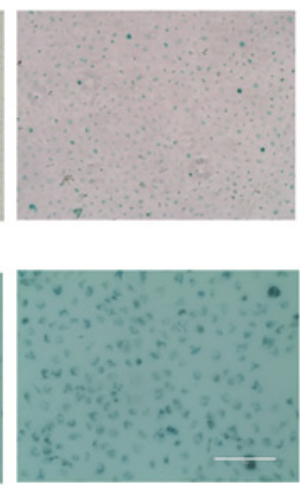

B
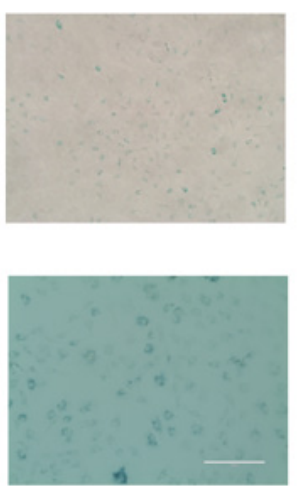

$\mathrm{C}$

Figure 1: SA- $\beta-$ Gal activity stain in four groups: A (blank control: $10 \%$ fetal bovine serum of F12 DMED); B (2\% fetal bovine serum of F12 DMEM); C (2\% fetal bovine serum of F12 DMEM + $20 \mu M$ icariin). We observed more SA- $\beta$-Gal activity stain in group $B$ and this activity stain have been attenuate.
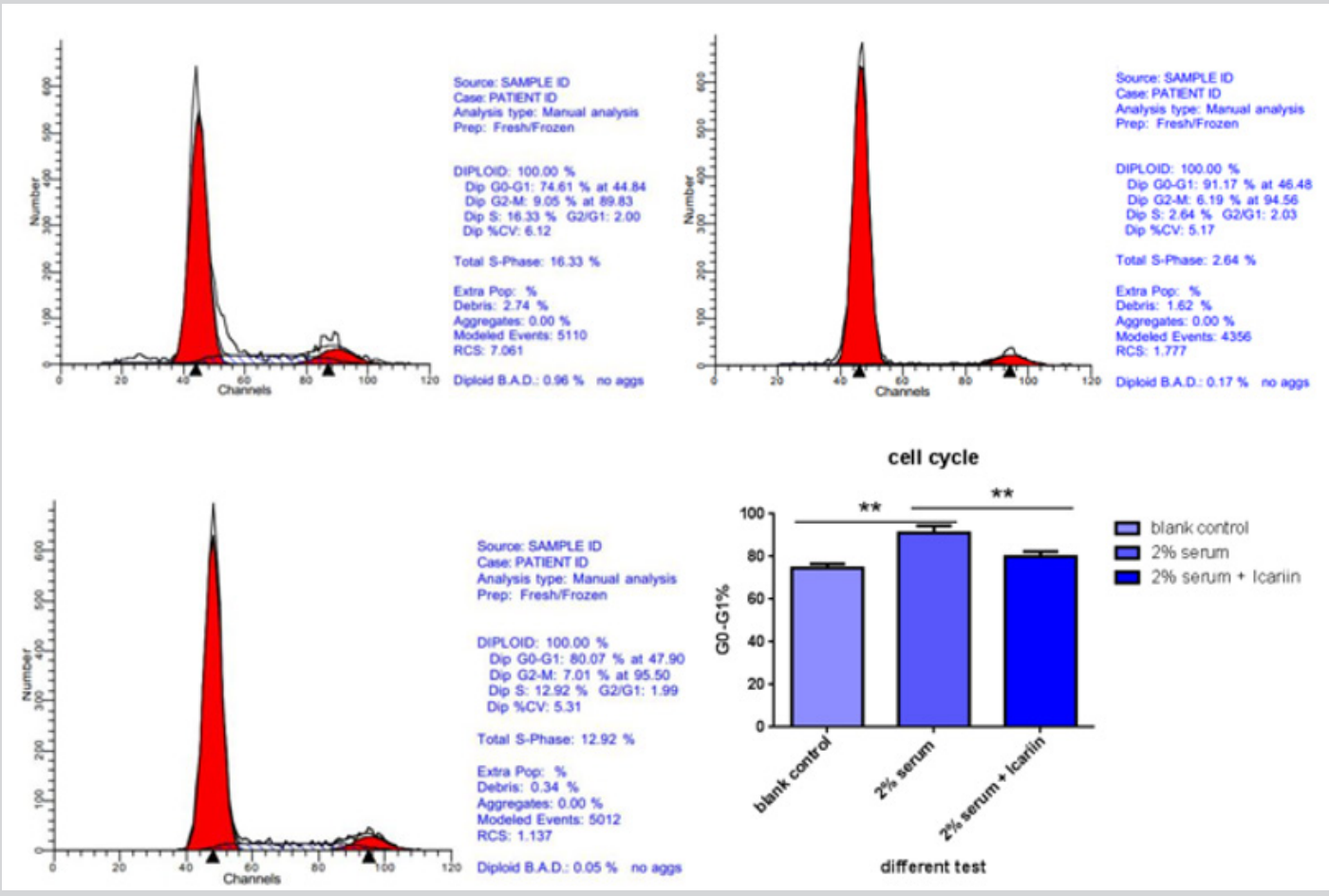

Figure 2: Icariin decreased the percentage of $G 1$ phase serum-deprived-treated annulus fibrous (AF) cells. AF cells were incubated with different test compounds for 48 hours. The percentage of $A F$ cells in the $G_{0} / G_{1}, G_{2} / M$ and $S$ phases in each group was shown in the respective images. There was statistic different in blank group and $2 \%$ serum group $(* p<0.05)$ but no different in $2 \%$ serum group and icariin group. Icariin only have little effect on cell cycle under this situation. 
(A)
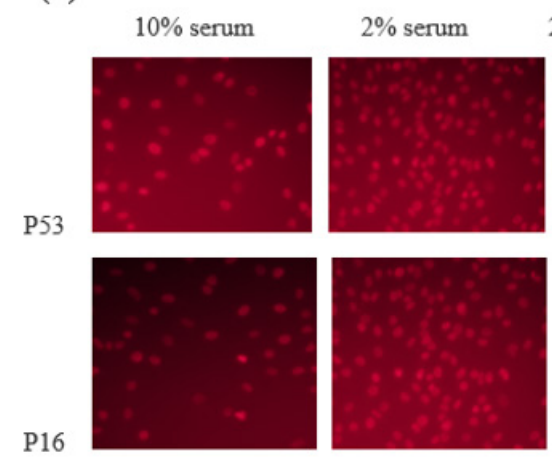

$2 \%$ serum+icariin
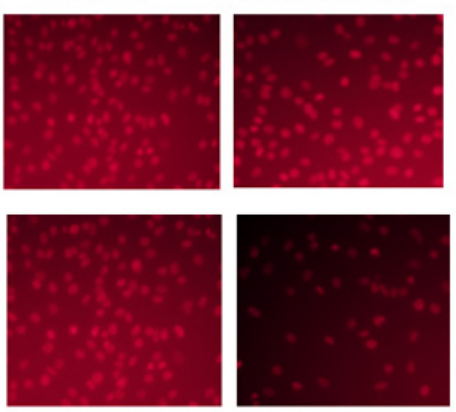

(B)

P53

P16

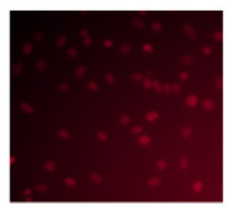

P53

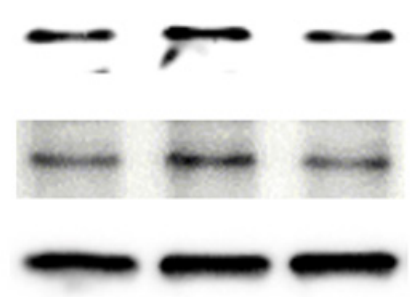

GAPDH

Serum $\quad 10 \% \quad 2 \% \quad 2 \%$

(C)

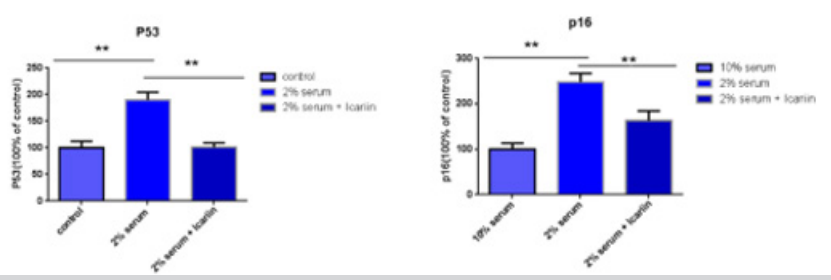

Figure 3: Compared with $10 \%$ serum group (blank group), $2 \%$ serum group showed up a significant increase in the expression of P53 and P16 $\left({ }^{*} \mathrm{p}<0.01\right)$, the two classic markers of cell enescence. These were supported by immunofluorescence in the cell (A) and western blot (B). Icariin decrease this protein expression and this data have significant statistic different $(* p<0.01)(C)$.

(A)

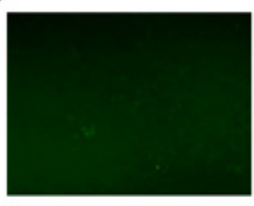

(B)

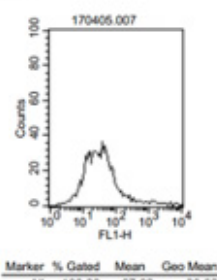

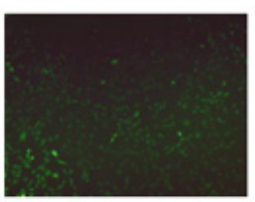

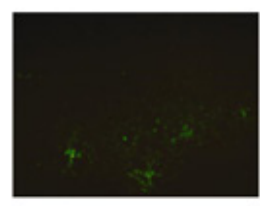

(C)

P-NF-kB/p-p65(ser 536)

GAPDH

(D)

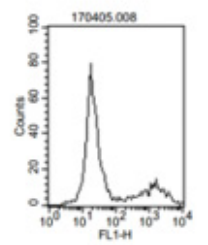

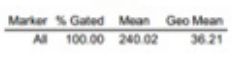

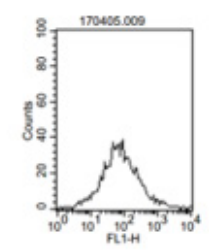

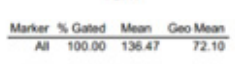
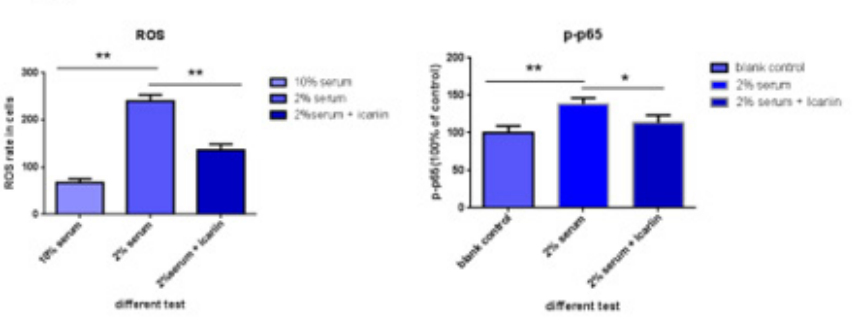

Figure 4: ROS rate rise remarkably when $10 \%$ serum became $2 \%$ serum indicate starvation induced oxidative stress in cells and NF-KB was activated and these were observed by intracellular immunofluorescence (A), flow cytometry (B) and western blot (C). Icariin can attenuate this effect a certain extent. This data has a significant statistic difference (D). $\left({ }^{*} p<0.05,{ }^{* *} p<0.01\right)$.

As showed in Figure 4, ROS/NF- $\kappa$ B pathway have a change in this serum-deprived model. ROS/NF- $\kappa B$ pathway is responsible for cell proliferate, apoptosis and survive. Continuously activated of ROS/NF- $\mathrm{BB}$ pathway have ability to induce senescence [22-24]. Here, we observed activation of ROS/NF-kB pathway when serumdeprived include the intercellular ROS rate rise by the methods of intracellular immunofluorescence (Figure 4A), flow cytometry (Figure 4B) and western blot (Figure 4C). The ascent of p-NF- $\kappa B$ (p-p65) and this activation of pathway was inhibition by icariin. This data has a significant statistic difference (Figure 4D). $\left({ }^{*} \mathrm{p}<0.05\right.$, ${ }^{* *} \mathrm{p}<0.01$ )

Icariin ameliorates disc degeneration in rat in vivo. As shown in Figure 5, the disc section was normal with round shape of the NP and well-organized collagen lamellae in the control group. However, in the IDD group, the disc appeared degenerative changes, and the 
histologic score was significantly higher than that of the control. Icariin treatment group improved the degenerative changes and decreased the histologic score. Furthermore, immunohistochemical staining showed that icariin could decreased expression levels of senescence markers p53 and up-regulated collagen I in AF cells as well as collagen II in NP cells, which confirmed our results in vitro.

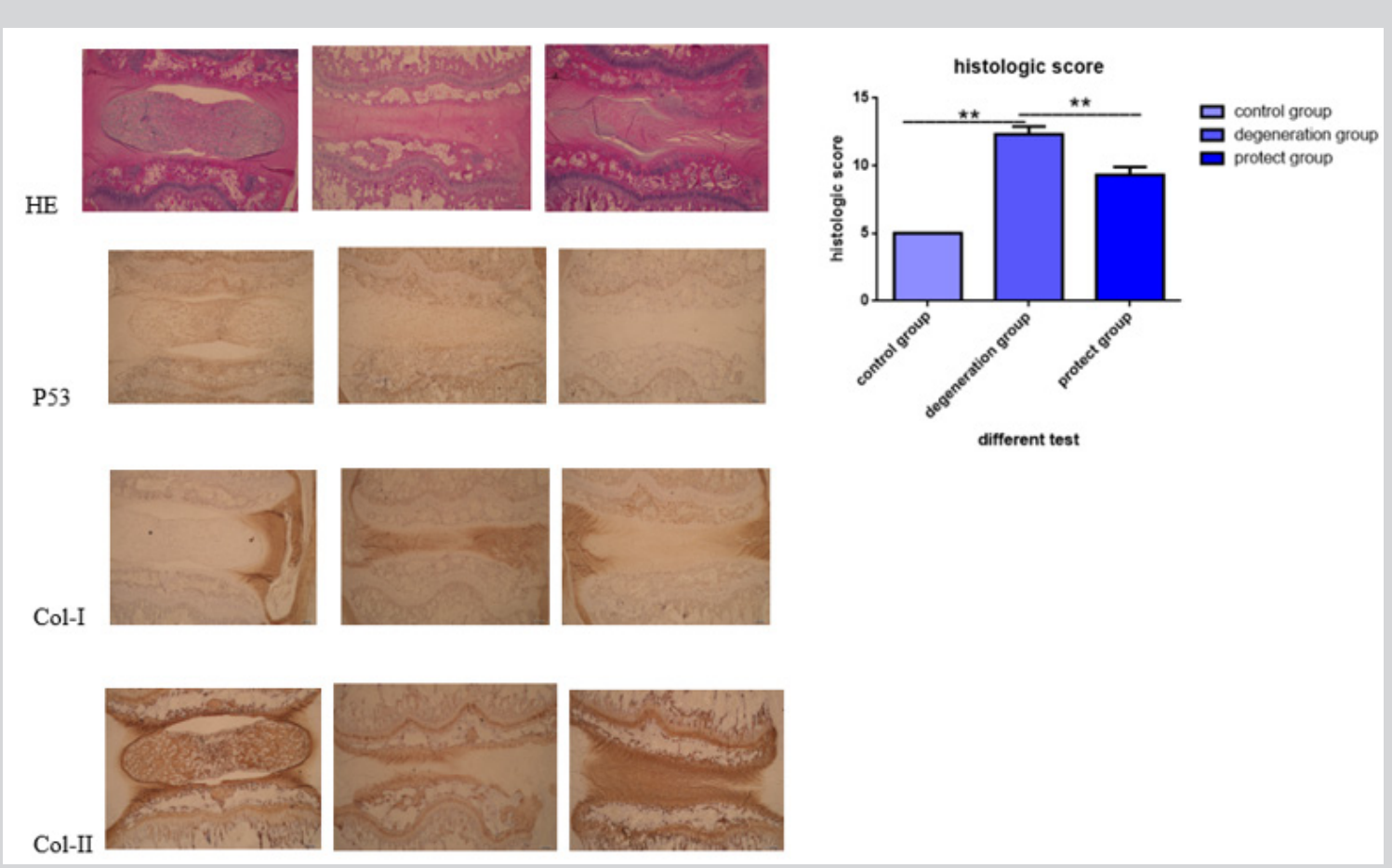

Figure 5: HE stains of intervertebral disc show we made the IVDD model successfully, histologic score demonstrated icariin have protect effect when rat nucleus pulposus tissue exposed to impaired fibrous rings tissue. Immunohistochemical of P53 showed fibrous rings aged when punctured by needle and icariin can protect against this aging process. Col-I and col-Il are the symbolic extracellular matrix (ECM) of intervertebral disc. We found icariin have active ability of deposition ECM, maintain the basic location of col-I and col-II, which can prevent the aging process of intervertebral disc.

\section{DISCUSSION}

Cellular senescence, which features irreversible growth arrest, can be induced by continuous replication and various stresses $[26,27]$. Under the adverse microenvironment, including pressure, hypoxia, nutrient deprivation, or reactive oxygen species, IVD cells senescence and apoptosis can accumulate in degenerative IVD [28]. Delaying IVD cell senescence may contribute to the IVDD repairment.

Our previous research indicated that icariin could protect against $\mathrm{H}_{2} \mathrm{O}_{2}$-induced apoptosis in rat nucleus pulposus cells, and it was reported that icariin is relative to aging $[29,30]$. However, there is no research discussing the effect of icariin on delaying senescence in intervertebral disc. We chose serum-deprived model to simulate the microenvironment of intervertebral disc for alimentary deficiency is an important factor for intervertebral disc degeneration [31]. After treatment of serum-deprived for $48 \mathrm{~h}$, we observed significant difference of cell premature senescence and these results are reflected by SA- $\beta$-gal stain, cell cycling detection, senescence relative proteins. Among these, SA- $\beta$-gal stain is used most wide for it is convenient and visual. Our results support that $\beta$-galactosidase expressed more when serum-deprived and icariin rescue this change. cell cycling detection had significant statistical significance in group B and C. As previous literature reported [32], in normal growth environment, annulus fibrosis cells have approximate $70 \%-80 \%$ G0-G1 cells. In our work, we got similar conclusion. And after $48 \mathrm{~h}$ serum-deprived treatment, almost all cells got into "period of dormancy" to adapt this unfavorable environment. We are amazed that icariin turns the scale to almost normal status. Our date gave the evidence that icariin has potential effect to restraint senescence. Whar's more, senescence relative protein p53 and p16 had same results. Nevertheless, target spot of icariin to play this role required more research.

For explore possible mechanism in this protect effect, we detect ROS/NF- $\kappa$ B pathway for icariin have strong effect to anti oxidative stress in previous research [33-37] and icariin may have effect on

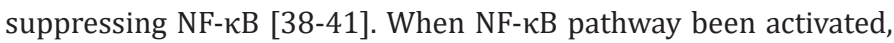
it's subunit $\mathrm{p} 65$ phosphorylate and get into cell nucleus to regulate transcription. So, detection of p-p65 is accurate to reflect state of NF- $\kappa$ B pathway. In group $B$, we observed remarkable change in intracellular ROS, an activating factor of NF- $\kappa B$, accompanied by increase of p-p65. When icariin pre-treatment, R0S and p-p65 decreased together. We believed that ROS/NF- $\kappa B$ pathway is at least partly involved in this protect effect of icariin. Certainly, more research is required to find more precise target spot, for icariin may be a potential medicine to treat IDD and delay senescence.

To evaluate the effect of icariin in vivo, a disc degeneration rat model was established and assessed. According to our histological analyses, icariin can prevent intervertebral disc from aging. The AF cells showed decreased expression levels of senescence markers (p53), up-regulated matrix protein expression and maintain the 
location distribution of collagen in intervertebral disc tissue in icariin treated group. All these results were in line with those from the AF cell culture in vitro.

\section{CONCLUSION}

In conclusion, Cell senescence induced by serum deprivation may contribute to AF degeneration and, thereby, disrupts IVD homeostasis. The results of this study indicated that icariin attenuate serum deprived-induced premature senescence in rat annulus fibrosus cells via ROS/NF- $\mathrm{BB}$ pathway and ameliorate disc degeneration in vivo. Icariin may by developed as a new therapeutic candidate for treatment of IVDD.

\section{REFERENCES}

1. Fontana G, See E, Pandit A (2015) Current trends in biologics delivery to restore intervertebral disc anabolism. Adv Drug Deliv Rev 84: 146-158.

2. Maniadakis N, Gray A (2000). The economic burden of back pain in the UK. Pain 84(1): 95-103.

3. Hwang MH, Shin JH, Kim KS (2015) Low-level light therapy modulates inflammatory mediators secreted by human annulus fibrosus cells during intervertebral disc degeneration in vitro. Photochem Photobiol 91(2): 403-410.

4. Stewart DM, Monaco LA, Gregory DE (2017) The aging disc: using an ovine model to examine age-related differences in the biomechanical properties of the interlamellar matrix of single lamellae. Eur Spine 26(1): 259-266.

5. Sarath BN, Krishnan S, Brahmendra SCV, Venkata SGP, Gurava RAV, et al. (2016) Quantitative proteomic analysis of normal and degenerated human intervertebral disc. Spine J 16(8): 989-1000.

6. Menezes-Reis R, Salmon CE, Bonugli GP (2016) Lumbar intervertebral discs $\mathrm{T} 2$ relaxometry and $\mathrm{T} 1 \rho$ relaxometry correlation with age in asymptomatic young adults. Quant Imaging Med Surg 6(4): 402-412.

7. Jiang LB, Liu HX, Zhou YL, Sheng SR, Xu HZ, et al. (2017) An ultrastructural study of chondroptosis: programmed cell death in degenerative intervertebral discs in vivo. J Anat.

8. Meng X, Pei H, Lan C (2015) Icariin Exerts Protective Effect Against Myocardial Ischemia/Reperfusion Injury in Rats. Cell Biochem Biophys 73(1): 229-235.

9. Zhai M, He L, Ju X, et al. (2015) Icariin Acts as a Potential Agent for Preventing Cardiac Ischemia/Reperfusion Injury. Cell Biochem Biophys 72(2): 589-597.

10. Yang D, Wang D, Shimer A, Shen FH, Li X, et al. (2014) Glutathione protects human nucleus pulposus cells from cell apoptosis and inhibition of matrix synthesis. Connect Tissue Res. 55(2): 132-139.

11. Xu CQ, Liu BJ, Wu JF, et al. (2010) Icariin attenuates LPS-induced acute inflammatory responses: involvement of PI3K/Akt and NF-kappa signalling pathway. Eur J Pharmocol 642(1-3): 146-153.

12. Zhang SQ, Cai WJ, Huang JH (2015) Icariin, a natural flavonol glycoside, extends healthspan in mice. Exp Gerontol 69: 226-235.

13. Schlesinger JK, Schlesinger H (2014) Plant polyphenols in the treatment of age-associated diseases: revealing the pleiotropic effects of icariin by network analysis. Mol Nutr Food Res 58(1): 49-60.

14. Wu B, Chen Y, Huang J, et al. (2012) Icariin improves cognitive deficits and activates quiescent neural stem cells in aging rats. J Ethnopharmacology 142(3): 746-753.

15. He XL, Zhou WQ Bi MG, Du GH (2010) Neuroprotective effects of icariin on memory impairment and neurochemical deficits in senescenceaccelerated mouse prone 8 (SAMP8) mice. Brain Res 1334: 73-83.

16. Chen Y, Sun T, Wu J, (2015) Icariin intervenes in cardiac inflammaging through upregulation of SIRT 6 enzyme activity and inhibition of the NFkappa B pathway. Biomed Res Int: 895976.
17. Xiao-Hong D, Chang-Qin X, Jian-Hua H, Wen-Jiang Z (2013) Bing S. Icariin delays homocysteine-induced endothelial cellular senescence involving activation of the PI3K/AKT-eNOS signaling pathway. Pharm Biol 51(4): 433-440.

18. Zhang Z, Zhang T, Dong K (2012) Icariin upregulates phosphorylated cyclic adenosine monophosphate response element binding protein levels in the hippocampus of the senescence- accelerated mouse. Neural Regen Res 7(12): 885-890.

19. Uemura Y, Kobayashi M, Nakata H, Harada R, Kubota T, et al. (2004) Effect of serum deprivation on constitutive production of granulocyte-colony stimulating factor and granulocyte macrophage-colony stimulating factor in lung cancer cells. Int J Cancer 109(6): 826-832.

20. Mogi M, Ozeki N, Nakamura H, Togari A (2004) Dual roles for NF-kappaB activation in osteoblastic cells by serum deprivation: osteoblastic apoptosis and cell-cycle arrest. Bone 35(2): 507-516.

21. Liu J, Du L (2015) PERK pathway is involved in oxygen-glucose-serum deprivation-induced NF-kB activation via ROS generation in spinal cord astrocytes. Biochem Biophys Res Commun 467(2): 197-203.

22. Zhang N, Li Z, Mu W (2016) Calorie restriction-induced SIRT6 activation delays aging by suppressing NF- $\kappa B$ signaling. Cell Cycle 15(7): 10091018.

23. Engelmann C, Haenold R (2016) Transcriptional control of synaptic plasticity by transcription factor NF- $\kappa$ B. Neural Plast: 7027949.

24. Jeong JW, Lee HH, Lee KW, (2016) Mori folium inhibits interleukin-1ßinduced expression of matrix metalloproteinases and inflammatory mediators by suppressing the activation of NF- $\kappa B$ and p38 MAPK in SW1353 human chondrocytes. Int J Mol Med 37(2): 452-460.

25. Chen D, Xia D, Pan Z (2016) Metformin protects against apoptosis and senescence in nucleus pulposus cells and ameliorates disc degeneration in vivo. Cell Death Dis 7(10): e2441.

26. Mao HJ, Chen QX, Han B (2011) The effect of injection volume on disc degeneration in a rat tail model. Spine (Phila Pa 1976) 36(16): E1062-9.

27.Zhou N, Lin X, Dong W (2016) SIRT1 alleviates senescence of degenerative human intervertebral disc cartilage endo-plate cells via the p53/p21 pathway. Sci Rep 6: 22628.

28. Wang F, Cai F, Shi R, Wang XH, Wu XT (2016) Aging and age-related stresses: a senescence mechanism of intervertebral disc degeneration. Osteoarthritis Cartilage 24(3): 398-408.

29. Gao L, Tang Q He X, Bi M (2012) Effect of icariin on learning and memory abilities and activity of cholinergic system of senescence-accelerated mice SAMP10. Zhongguo Zhong Yao Za Zhi 37(14): 2117-21.

30. Ye LC, Chen JM (2001) Advances in study on pharmacological effects of Epimedium. Zhongguo Zhong Yao Za Zhi 26(5): 293-5.

31. Johnson WE, Stephan S, Roberts S (2008) The influence of serum, glucose and oxygen on intervertebral disc cell growth in vitro: implications for degenerative disc disease. Arthritis Res Ther 10(2): R46.

32. Wang F, Cai F, Shi R, Wei JN, Wu XT (2016) Hypoxia regulates sumoylation pathways in intervertebral disc cells: implications for hypoxic adaptations. Osteoarthritis Cartilage 24(6): 1113-11124.

33. Tang Y, Jacobi A, Vater C, Zou L, Zou X, Stiehler M , et al.( 2015)Icariin promotes angiogenic differentiation and prevents oxidative stressinduced autophagy in endothelial progenitor cells. Stem Cells 33(6): 1863-1877.

34. Wang YK, Huang ZQ (2005) Protective effects of icariin on human umbilical vein endothelial cell injury induced by $\mathrm{H}_{2} \mathrm{O}_{2}$ in vitro. Pharmacol Res 52(2): 174-182.

35. Wu J, Xu H, Wong PF, Xia S, Xu J, et al. (2014) Icaritin attenuates cigarette smoke-mediated oxidative stress in human lung epithelial cells via activation of PI3K-AKT and Nrf2 signaling. Food Chem Toxicol 64: 307313.

36. Zhang L, Huang S, Chen Y, Wang Z, Li E, et al. (2010) Icariin inhibits hydrogen peroxide-mediated cytotoxicity by up-regulating sirtuin type 
1-dependent catalase and peroxiredoxin. Basic Clin Pharmacol Toxicol 107(5): 899-905.

37. Ma HP, Ma XN, Ge BF (2014) Icariin attenuates hypoxia-induced oxidative stress and apoptosis in osteoblasts and preserves their osteogenic differentiation potential in vitro. Cell Prolif 47(6): 527-539.

38. Zhang DC, Liu JL, Ding YB, Xia JG, Chen GY, et al. (2013) Icariin potentiates the antitumor activity of gemcitabine in gallbladder cancer by suppressing NF-кB. Acta Pharmacol Sin 34(2): 301-308.

39. Li L, Sun J, Xu C, et al. (2014) Icariin ameliorates cigarette smoke induced inflammatory responses via suppression of NF- $\mathrm{\kappa B}$ and modulation of GR in vivo and in vitro. PLoS One 9(8): e102345.

40. Hsieh TP, Sheu SY, Sun JS, Chen MH (2011) Icariin inhibits osteoclast differentiation and bone resorption by suppression of MAPKs/NF-kB regulated HIF-1 $\alpha$ and PGE (2) synthesis. Phytomedicine 18(2-3): 17685.

41.Zeng KW, Fu H, Liu GX, Wang XM (2010) Icariin attenuates lipopolysaccharide-induced microglial activation and resultant death of neurons by inhibiting TAK1/IKK/NF-kappaB and JNK/p38 MAPK pathways. Int Immunopharmacol 10(6): 668-678. 